\title{
NON SELF-ADJOINT LAPLACIANS ON A DIRECTED GRAPH
}

\author{
MARWA BALTI
}

\begin{abstract}
We consider a non self-adjoint Laplacian on a directed graph with non symmetric edge weights. We analyse spectral properties of this Laplacian under a Kirchhoff assumption. Moreover we establish isoperimetric inequalities in terms of the numerical range to show the absence of the essential spectrum of the Laplacian on heavy end directed graphs.
\end{abstract}

\section{Contents}

Introduction

1. Preliminaries

1.1. Notion of Graphs

1.2. Functional spaces

1.3. Laplacian on a directed graph

2. Spectral analysis of the bounded case

3. Spectral study of the unbounded case

3.1. Closable operator

3.2. Cheeger inequalities

3.3. Absence of essential spectrum from Cheeger constant

References

\section{INTRODUCTION}

The non self-adjoint operators are more difficult to study than the self-adjoint ones: no spectral theorem in general, wild resolvent growth... The related theory is studied by different authors: L. N. Trefethen [Tr05] for non symmetric matrices, W. D. Evans, R. T. Lewis, A. Zettl ELZ83 and R. T. Lewis Lew79] for non self-adjoint operators in a Hilbert space. Recently, the interest in spectral properties of non self-adjoint operators has already led to a variety of new results, both in the continuous and discrete settings, e.g, bounds on complex eigenvalues [FLS11] and Lieb-Thirring type inequalities [Han11, [DHK09]. This can be explained by the complicated structure of the resolvent of such an operator seen as an analytic function. In this paper we focus on directed graphs to study a non symmetric Laplacian. We develop a general approximation theory

2010 Mathematics Subject Classification. 47A45, 47A12, 47A10, 47B25.

Key words and phrases. Directed graph, Graph Laplacian, Non self-adjoint operator, Numerical range, Eigenvalues, Essential spectrum. 
for the eigenvalues on directed graphs with non symmetric edge weights assuming only a condition of "total conductivity of the vertices" presented as the Assumption $(\beta)$. We investigate the spectrum of our discrete non self-adjoint Laplacian. We collect some basic properties of the Laplacian and we seek to show the emptiness of its essential spectrum by using isoperimetric inequalities. We explain how isoperimetric inequalities can be linked to the numerical range of non symmetric operators. In fact, for the self-adjoint Laplace-Beltrami operator, Jeff Cheeger proved an inequality that links the first nontrivial eigenvalue on a compact Riemannian manifold to a geometric constant $h$. This inspired an analogous theory for graphs (see [Fuj96], Gri11]). In this work, we introduce a kind of Cheeger constant on a filtration of a directed graph $G$ and we estimate the associated Laplacian $\Delta$. We give an estimation for the numerical range of $\Delta$ in terms of the Cheeger constant. We use this estimation and propose a condition on the weights for the absence of essential spectrum of heavy end directed graphs. There is an analogous result of H. Donnelly and P. Li [DL79] for a self-adjoint operator on complete negatively curved manifolds. They show that the Laplacian on a rapidly curving manifold has a compact resolvent.

Section 1 is devoted to some definitions and notions on a directed graph with non symmetric edge weights and the associate non symmetric differential Laplacian. We describe some basic results: Green's formula and the spectral properties of $\Delta$ and of its formal adjoint.

In Section 2, we study spectral properties of the bounded operator $\tilde{\Delta}$ by relying on known results for the symmetric case.

In Section 3, we establish the Cheeger inequality for the non symmetric Dirichlet Laplacian on any subset of the set of vertices $V$ to give a lower bound for the bottom of the real part of the numerical range. We control the real part of the numerical range of $\Delta$ and relate it with the spectrum of the its closure $\bar{\Delta}$. We characterize the absence of essential spectrum of $\bar{\Delta}$. Fujiwara Fuj96 and Keller [Kel10] introduced a criterion for the absence of essential spectrum of the symmetric Laplacian on a rapidly branching graph. In fact, our criterion is: positivity of the Cheeger constant at infinity on heavy end graphs.

\section{Preliminaries}

We review in this section some basic definitions on infinite weighted graphs and introduce the notation used in the article. They are introduced in Bal16] for finite non symmetric graphs (see [AT15] and [T-H10] for the symmetric case).

1.1. Notion of Graphs. A directed weighted graph is a triplet $G:=(V, \vec{E}, b)$, where $V$ is a countable set (the vertices), $\vec{E}$ is the set of directed edges and $b: V \times V \rightarrow[0, \infty)$ is a weight function satisfying the following conditions:

- $b(x, x)=0$ for all $x \in V$

- $b(x, y)>0$ iff $(x, y) \in \vec{E}$

In addition, we consider a measure on $\mathrm{V}$ given by a positive function

$$
m: V \rightarrow(0, \infty) \text {. }
$$


The weighted graph is symmetric if for all $x, y \in V, b(x, y)=b(y, x)$, as a consequence $(x, y) \in \vec{E} \Leftrightarrow(y, x) \in \vec{E}$.

The graph is called simple if the weights $m$ and $b$ are constant and equal to 1 on $V$ and $\vec{E}$ respectively.

The set $E$ of undirected edges is given by

$$
E=\{\{x, y\},(x, y) \in \vec{E} \text { or }(y, x) \in \vec{E}\} .
$$

Definition 1.1. Define for a subset $\Omega$ of $V$, the vertex boundary and the edge boundary of $\Omega$ respectively by:

$$
\begin{gathered}
\partial_{V} \Omega=\left\{y \in \Omega:\{x, y\} \in E \text { for some } x \in \Omega^{c}\right\} \\
\partial_{E} \Omega=\left\{(x, y) \in \vec{E}:\left(x \in \Omega, y \in \Omega^{c}\right) \text { or }\left(x \in \Omega^{c}, y \in \Omega\right)\right\} .
\end{gathered}
$$

On a non symmetric graph we have two notions of connectedness.

Definition 1.2. $\quad$ - A path between two vertices $x$ and $y$ in $V$ is a finite set of directed edges $\left(x_{1}, y_{1}\right) ;\left(x_{2}, y_{2}\right) ; . . ;\left(x_{n}, y_{n}\right), n \geq 2$ such that

$$
x_{1}=x, y_{n}=y \text { and } x_{i}=y_{i-1} \quad \forall 2 \leq i \leq n
$$

- $G$ is called connected if two vertices are always related by a path.

- $G$ is called strongly connected if for all vertices $x, y$ there is a path from $x$ to $y$ and one from $y$ to $x$.

1.2. Functional spaces. Let us introduce the following spaces associated to the graph $G$ :

- the space of functions on the graph $G$ is considered as the space of complex functions on $\mathrm{V}$ and is denoted by

$$
\mathcal{C}(V)=\{f: V \rightarrow \mathbb{C}\}
$$

- $\mathcal{C}_{c}(V)$ is its subset of finite supported functions;

- we consider for a measure $m$, the space

$$
\ell^{2}(V, m)=\left\{f \in \mathcal{C}(V), \quad \sum_{x \in V} m(x)|f(x)|^{2}<\infty\right\} .
$$

It is a Hilbert space when equipped by the scalar product given by

$$
(f, g)_{m}=\sum_{x \in V} m(x) f(x) \overline{g(x)} .
$$

The associated norm is given by:

$$
\|f\|_{m}=\sqrt{(f, f)_{m}} .
$$


1.3. Laplacian on a directed graph. In this work, we assume that the graph under consideration is connected, locally finite, without loops and satisfies for all $x \in V$ the following conditions:

$$
\sum_{y \in V} b(x, y)>0 \text { and } \sum_{y \in V} b(y, x)>0 .
$$

We introduce the combinatorial Laplacian $\Delta$ defined on $\mathcal{C}_{c}(V)$ by:

$$
\Delta f(x)=\frac{1}{m(x)} \sum_{y \in V} b(x, y)(f(x)-f(y)) .
$$

For all $x \in V$ we note by $\beta^{+}(x)=\sum_{y \in V} b(x, y)$, in particular if $m(x)=\beta^{+}(x)$ then the Laplacian is said to be the normalized Laplacian and it is defined by:

$$
\tilde{\Delta} f(x)=\frac{1}{\beta^{+}(x)} \sum_{y \in V} b(x, y)(f(x)-f(y)) .
$$

Dirichlet operator: Let $U$ be a subset of $V, f \in \mathcal{C}_{c}(U)$ and $g: V \rightarrow \mathbb{C}$ the extension of $f$ to $V$ by setting $g=0$ outside $U$. For any operator $A$ on $\mathcal{C}_{c}(V)$, the Dirichlet operator $A_{U}^{D}$ is defined by

$$
A_{U}^{D}(f)=\left.A(g)\right|_{U}
$$

The operator $\Delta$ may be non symmetric if the edge weight is not symmetric.

Proposition 1.1. The formal adjoint $\Delta^{\prime}$ of the operator $\Delta$ is defined on $\mathcal{C}_{c}(V)$ by:

$$
\Delta^{\prime} f(x)=\frac{1}{m(x)}\left(\sum_{y \in V} b(x, y) f(x)-\sum_{y \in V} b(y, x) f(y)\right)
$$

Proof:

For all $f, g \in \mathcal{C}_{c}(V)$, we have

$$
\begin{aligned}
(\Delta f, g)_{m} & =\sum_{(x, y) \in \vec{E}} b(x, y)(f(x)-f(y)) \overline{g(x)} \\
& =\sum_{x \in V} f(x) \sum_{y \in V} b(x, y) \overline{g(x)}-\sum_{(y, x) \in \vec{E}} b(y, x) \overline{g(y)} f(x) \\
& =\sum_{x \in V} f(x)\left(\sum_{y \in V} b(x, y) \overline{g(x)}-\sum_{(y, x) \in \vec{E}} b(y, x) \overline{g(y)}\right) .
\end{aligned}
$$

As $(\Delta f, g)_{m}=\left(f, \Delta^{\prime} g\right)_{m}$, so we get

$$
\Delta^{\prime} f(x)=\frac{1}{m(x)}\left(\sum_{y \in V} b(x, y) f(x)-\sum_{y \in V} b(y, x) f(y)\right) .
$$


Remark 1.1. The operator $\Delta^{\prime}$ can be expressed as a Schrödinger operator with the potential $q(x)=\frac{1}{m(x)} \sum_{y \in V}(b(x, y)-b(y, x)), x \in V$ :

$$
\Delta^{\prime} f(x)=\frac{1}{m(x)} \sum_{y \in V} b(y, x)(f(x)-f(y))+q(x) f(x) .
$$

We introduce here the Assumption $(\beta)$ and we assume that it is satisfied by the considered weighted graph, throughout the rest.

Assumption $(\beta)$ : for all $x \in V, \beta^{+}(x)=\beta^{-}(x)$ where

$$
\beta^{+}(x)=\sum_{y \in V} b(x, y) \text { and } \beta^{-}(x)=\sum_{y \in V} b(y, x) .
$$

Remark 1.2. The Assumption $(\beta)$ is natural. Indeed, it looks like the Kirchhoff's law in the electrical networks.

Corollary 1.1. We suppose that the Assumption $(\beta)$ is satisfied, the operator $\Delta^{\prime}$ is simply a Laplacian, given by

$$
\Delta^{\prime} f(x)=\frac{1}{m(x)} \sum_{y \in V} b(y, x)(f(x)-f(y)) .
$$

In the sequel, for the sake of simplicity we introduce the symmetric Laplacian $H$ associated to the graph with the symmetric edge weight function $a(x, y)=$ $b(x, y)+b(y, x)$. It acts on $\mathcal{C}_{c}(V)$ by,

$$
H f(x)=\left(\Delta+\Delta^{\prime}\right) f(x)=\frac{1}{m(x)} \sum_{y \in V} a(x, y)(f(x)-f(y)) .
$$

The quadratic form $Q_{\Delta}$ of $H$ is given by

$$
Q_{\Delta}(f)=(\Delta f, f)+\overline{(\Delta f, f)}, f \in \mathcal{C}_{c}(V) .
$$

Comment 1.1. Let $f \in \mathcal{C}_{c}(V)$, we have $Q_{\Delta}(f)=2 \mathcal{R} e(\Delta f, f)$. Then

$$
\inf _{\|f\|_{m}=1} Q_{\Delta}(f)=\inf _{\|f\|_{m}=1} 2 \mathcal{R} e(\Delta f, f) .
$$

We establish an explicit Green's formula associated to the non self-adjoint Laplacian $\Delta$ from which any estimates on the symmetric quadratic form $Q_{\Delta}$ can be directly cited from the literature.

Lemma 1.1. (Green's Formula) Let $f$ and $g$ be two functions of $\mathcal{C}_{c}(V)$. Then under the Assumption ( $\beta$ ) we have

$$
(\Delta f, g)_{m}+{\overline{(\Delta g, f)_{m}}}_{=}=\sum_{(x, y) \in \vec{E}} b(x, y)(f(x)-f(y))(\overline{g(x)-g(y)}) .
$$

Proof: 
The proof is given by a simple calculation. From Corollary 1.1, we have

$$
\begin{aligned}
(\Delta f, g)_{m}+{\overline{(\Delta g, f)_{m}}} & =(H f, g)_{m} \\
& =\sum_{(x, y) \in \vec{E}} b(x, y)(f(x)-f(y)) \overline{g(x)} \\
& +\sum_{(y, x) \in \vec{E}} b(y, x)(f(x)-f(y)) \overline{g(x)} \\
& =\sum_{(x, y) \in \vec{E}} b(x, y)(f(x) \overline{g(x)}+f(x) \overline{g(x)}-f(y) \overline{g(x)}-f(x) \overline{g(y)}) \\
& =\sum_{(x, y) \in \vec{E}} b(x, y)(f(x)-f(y))(\overline{g(x)-g(y)}) .
\end{aligned}
$$

We refer to Kat76 page 243 for the definitions of the spectrum and the essential spectrum of a closed operator $A$ in a Hilbert space $\mathcal{H}$, with domain $D(A)$.

Definition 1.3. - The spectrum $\sigma(A)$ of $A$ is the set of all complex numbers $\lambda$ such that $(A-\lambda)$ has no bounded inverse.

- The essential spectrum $\sigma_{\text {ess }}(A)$ of $A$ is the set of all complex numbers $\lambda$ for which the range $R(A-\lambda)$ is not closed or $\operatorname{dim} \operatorname{ker}(A-\lambda)=\infty$.

\section{Spectral analysis of the Bounded CASE}

This part concerns some basic properties of the bounded non self-adjoint Laplacian $\tilde{\Delta}$. We introduce the concept of the numerical range. It has been extensively studied the last few decades. This is because it is very useful in studying and understanding the spectra of operators (see [Ber64], JY12, [AZ10]).

Definition 2.1. The numerical range of an operator $T$ with domain $D(T)$, denoted by $W(T)$ is the non-empty set

$$
W(T)=\{(T f, f), \quad f \in D(T),\|f\|=1\} .
$$

The following Theorem in JY12] shows that the spectrum behave nicely with respect to the closure of the numerical range.

Theorem 2.1. Let $\mathcal{H}$ be a reflexive Banach space and $T$ a bounded operator on $\mathcal{H}$. Then:

$$
\sigma(T) \subset \overline{W(T)}
$$

The following Proposition is one of the main tools when working with the normalized Laplacian.

Proposition 2.1. Suppose that the Assumption $(\beta)$ is satisfied. Then $\tilde{\Delta}$ is bounded by 2 .

Proof: 


$$
\begin{aligned}
\mid(\tilde{\Delta} f, g)_{\beta^{+}} & =\left|\sum_{x \in V} \overline{g(x)} \sum_{y \in V} b(x, y)(f(x)-f(y))\right| \\
& \leq \sum_{x \in V} \beta^{+}(x)|f(x) \overline{g(x)}|+\sum_{x \in V}|\overline{g(x)}| \sum_{y \in V} b(x, y)|f(y)|
\end{aligned}
$$

by the Assumption $(\beta)$ and the Cauchy-Schwarz inequality we prove the result:

$$
\begin{aligned}
\mid(\tilde{\Delta} f, g)_{\beta^{+}} & \leq(f, f)_{\beta^{+}}^{\frac{1}{2}}(g, g)_{\beta^{+}}^{\frac{1}{2}}+\sum_{x \in V}|\overline{g(x)}|\left(\sum_{y \in V} b(x, y)\right)^{\frac{1}{2}}\left(\sum_{y \in V} b(x, y)|f(y)|^{2}\right)^{\frac{1}{2}} \\
& \leq(f, f)_{\beta^{+}}^{\frac{1}{2}}(g, g)_{\beta^{+}}^{\frac{1}{2}}+\left(\sum_{x \in V} \beta^{+}(x)|g(x)|^{2}\right)^{\frac{1}{2}}\left(\sum_{x \in V} \sum_{y \in V} b(x, y)|f(y)|^{2}\right)^{\frac{1}{2}} \\
& \leq(f, f)_{\beta^{+}}^{\frac{1}{2}}(g, g)_{\beta^{+}}^{\frac{1}{2}}+(g, g)_{\beta^{+}}^{\frac{1}{2}}\left(\sum_{y \in V}|f(y)|^{2} \sum_{x \in V} b(x, y)\right)^{\frac{1}{2}} \\
& \leq(f, f)_{\beta^{+}}^{\frac{1}{2}}(g, g)_{\beta^{+}}^{\frac{1}{2}}+(g, g)_{\beta^{+}}^{\frac{1}{2}}\left(\sum_{y \in V}|f(y)|^{2} \beta^{-}(y)\right)^{\frac{1}{2}} \\
& \leq 2(f, f)_{\beta^{+}}^{\frac{1}{2}}(g, g)_{\beta^{+}}^{\frac{1}{2}} .
\end{aligned}
$$

Then

$$
\|\tilde{\Delta}\|_{\beta^{+}}=\sup _{\substack{\|f\|_{\beta^{+}} \leq 1 \\\|g\|_{\beta^{+}} \leq 1}}\left|(\tilde{\Delta} f, g)_{\beta^{+}}\right| \leq 2 .
$$

It is useful to develop some basic properties of the numerical range to make the computations of the spectrum of the Laplacian.

Proposition 2.2. Let $G$ be a connected graph, satisfying the Assumption $(\beta)$. Then

(1) $\sigma(\tilde{\Delta}) \subset D(1,1)$, the closed disc with center $(1,0)$ and radius 1 .

(2) If $\beta^{+}(V)<\infty$, then 0 is a simple eigenvalue of $\tilde{\Delta}$.

Proof:

(1) By Cauchy-Schwarz inequality as in (2), for $f \in D(\Delta)$ we have

$$
\begin{aligned}
\left|(\tilde{\Delta} f, f)_{\beta^{+}}-(f, f)_{\beta^{+}}\right| & =\left|\sum_{x \in V} \sum_{y \in V} b(x, y) f(x) \overline{f(y)}\right| \\
& \leq \sum_{x \in V} \sum_{y \in V} b(x, y)|f(x) \overline{f(y)}| \\
& \leq(f, f)_{\beta^{+}}
\end{aligned}
$$

which implies that $W(\tilde{\Delta}) \subset D(1,1)$. 
(2) If $\sum_{x \in V} \beta^{+}(x)=\sum_{x \in V} \beta^{+}(x)<\infty$, the constant function is an eigenfunction of $\tilde{\Delta}$ associated to 0 . Then 0 is an eigenvalue of $\tilde{\Delta}$. Now, we suppose that $f$ is an eigenfunction of $\tilde{\Delta}$ associated to 0 , therefore $\left(\left(\tilde{\Delta}+\tilde{\Delta}^{\prime}\right) f, f\right)=0$. Thus by connectedness of $G, f$ is constant.

It is obvious that $\operatorname{Re} e(A)=\frac{1}{2}\left(A+A^{*}\right)$ if $A$ is a bounded operator, but this is not true in general. The result below establishes a link between the real part of a matrix and its eigenvalues considered as the roots of the characteristic polynomial, see GC05 page 8. The adjoint of a square matrix is the transpose of its conjugate.

Lemma 2.1. Let $A$ be a square matrix of size $n, \lambda_{k}(A)$ and $\lambda_{k}(\mathcal{R} e(A))$, $k=1, . ., n$ the eigenvalues of $A$ and $\mathcal{R} e(A)$ respectively. Suppose that the eigenvalues of $\mathcal{R} e(A)$ are labelled in the increasing order, so that, $\lambda_{1}(\mathcal{R} e(A)) \leq$ $\lambda_{2}(\mathcal{R} e(A)) . . \leq \lambda_{n}(\mathcal{R} e(A))$. Then

$$
\sum_{k=n-q+1}^{n} \mathcal{R} e\left(\lambda_{k}(A)\right) \leq \sum_{k=n-q+1}^{n} \lambda_{k}(\mathcal{R} e(A)), \quad \forall q=1, . ., n
$$

and the equality prevails for $q=n$.

Remark 2.1. It should be noted that for a matrix $A, \lambda_{k}(\mathcal{R} e(A))$ and $\mathcal{R} e\left(\lambda_{k}(A)\right)$ are not equal in general. We can see [Bal16] for a counter-example.

In the following we study some generalities of eigenvalues of $\tilde{\Delta}_{\Omega}^{D}$, where $\Omega$ is a finite subset of $V$. We assume that they are ordered as follows:

$$
\mathcal{R} e\left(\lambda_{1}\left(\tilde{\Delta}_{\Omega}^{D}\right)\right) \leq \mathcal{R} e\left(\lambda_{2}\left(\tilde{\Delta}_{\Omega}^{D}\right)\right) . . \leq \mathcal{R} e\left(\lambda_{n}\left(\tilde{\Delta}_{\Omega}^{D}\right)\right) .
$$

Lemma 2.2. Let $\Omega$ be a finite non-empty subset of $V$, we have

$$
\lambda_{1}\left(\mathcal{R} e\left(\tilde{\Delta}_{\Omega}^{D}\right)\right) \leq \mathcal{R} e\left(\lambda_{1}\left(\tilde{\Delta}_{\Omega}^{D}\right)\right) .
$$

Proof:

Let $f$ be an eigenfunction associated to $\lambda_{1}\left(\tilde{\Delta}_{\Omega}^{D}\right)$. By the variational principle of $\lambda_{1}\left(\tilde{H}_{\Omega}^{D}\right)$, we have

$$
\begin{aligned}
\lambda_{1}\left(\tilde{H}_{\Omega}^{D}\right) & \leq \frac{\left(\tilde{H}_{\Omega}^{D} f, f\right)_{m}}{(f, f)_{m}} \\
& =\frac{\left(\tilde{\Delta}_{\Omega}^{D} f, f\right)_{m}}{(f, f)_{m}}+\frac{\overline{\left(\tilde{\Delta}_{\Omega}^{D} f, f\right)_{m}}}{(f, f)_{m}} \\
& =\lambda_{1}\left(\tilde{\Delta}_{\Omega}^{D}\right)+\overline{\lambda_{1}\left(\tilde{\Delta}_{\Omega}^{D}\right)} .
\end{aligned}
$$

The next statement contains an additional information about the eigenvalues of $\tilde{\Delta}_{\Omega}^{D}$.

Proposition 2.3. Let $\Omega$ be a finite non-empty subset of $V(\# \Omega=n)$ such that $\partial_{V} \Omega \neq \emptyset$. Then the following assertions are true 
(1) $0<\mathcal{R} e\left(\lambda_{1}\left(\tilde{\Delta}_{\Omega}^{D}\right)\right) \leq 1$.

(2) $\lambda_{1}\left(\mathcal{R} e\left(\tilde{\Delta}_{\Omega}^{D}\right)\right)+\lambda_{n}\left(\mathcal{R} e\left(\tilde{\Delta}_{\Omega}^{D}\right)\right) \leq 2$.

Proof:

(1) From Theorem 4.3 of [Gri11, we have $\lambda_{1}\left(\mathcal{R} e\left(\tilde{\Delta}_{\Omega}^{D}\right)\right)>0$ and by Lemma 2.2 we conclude the left inequalty. Next, by Lemma 2.1 we have for $q=n$ :

$$
\sum_{k=1}^{n} \mathcal{R} e\left(\lambda_{k}\left(\tilde{\Delta}_{\Omega}^{D}\right)\right)=\sum_{k=1}^{n} \lambda_{k}\left(\mathcal{R} e\left(\tilde{\Delta}_{\Omega}^{D}\right)\right.
$$

then

$$
n \mathcal{R} e\left(\lambda_{1}\left(\tilde{\Delta}_{\Omega}^{D}\right)\right) \leq \sum_{k=1}^{n} \lambda_{k}\left(\mathcal{R} e\left(\tilde{\Delta}_{\Omega}^{D}\right)=\operatorname{Tr}\left(\mathcal{R} e\left(\tilde{\Delta}_{\Omega}^{D}\right)\right)=n\right.
$$

which proves that

$$
\mathcal{R} e\left(\lambda_{1}\left(\tilde{\Delta}_{\Omega}^{D}\right)\right) \leq 1
$$

(2) It is deduced from the result of the symmetric case, see Theorem 4.3 [Gri11.

Corollary 2.1. Let $\Omega$ be a finite non-empty subset of $V$, then

$$
\mathcal{R} e\left(\lambda_{n}\left(\tilde{\Delta}_{\Omega}^{D}\right)\right)<2 .
$$

Proof:

Applying the Lemma 2.1 for $q=1$, we get

$$
\mathcal{R} e\left(\lambda_{n}\left(\tilde{\Delta}_{\Omega}^{D}\right)\right) \leq \lambda_{n}\left(\mathcal{R} e\left(\tilde{\Delta}_{\Omega}^{D}\right)\right) .
$$

But by (2) of Proposition 2.3, we have :

$$
\lambda_{n}\left(\mathcal{R} e\left(\tilde{\Delta}_{\Omega}^{D}\right)\right) \leq 2-\lambda_{1}\left(\mathcal{R} e\left(\tilde{\Delta}_{\Omega}^{D}\right)\right) .
$$

Then from the general property $\lambda_{1}\left(\mathcal{R} e\left(\tilde{\Delta}_{\Omega}^{D}\right)\right)>0$, we conclude that $\lambda_{n}\left(\mathcal{R} e\left(\tilde{\Delta}_{\Omega}^{D}\right)\right)<2$.

\section{Spectral study of the unbounded case}

This part includes the study of the bounds on the numerical range and the essential spectrum of a closed Laplacian. Both issues can be approached via isoperimetric inequalities. 
3.1. Closable operator. The purpose of the theory of unbounded operators is essentially to construct closed extensions of a given operator and to study their properties.

Definition 3.1. Closable operators: A linear operator $T: D(T) \rightarrow \mathcal{H}$ is closable if it has closed extensions.

An interesting property for the Laplacian $\Delta$ is its closability.

Proposition 3.1. Let $G$ be a graph satisfying the Assumption $(\beta)$. Then $\Delta$ is a closable operator.

Proof:

We shall use the Theorem of T. Kato which says that an operator densely defined is closable if its numerical range is not the whole complex plane, see [Kat76], page 268. Let $\lambda \in W(\Delta)$, there is $f \in \mathcal{C}_{c}(V)$ such that $\|f\|_{m}=1$ and $\lambda=(\Delta f, f)_{m}$. From the Green's formula we have,

$$
2 \mathcal{R} e(\lambda)=\sum_{(x, y) \in \vec{E}} b(x, y)|f(x)-f(y)|^{2} \geq 0 .
$$

It follows that $W(\Delta) \subset\{\lambda \in \mathbb{C}, \quad \mathcal{R} e(\lambda) \geq 0\} \subsetneq \mathbb{C}$.

For such operators, another property of interest is the property of being closed.

Definition 3.2. The closure of $\Delta$ is the operator $\bar{\Delta}$, defined by

- $D(\bar{\Delta})=\left\{f \in \ell^{2}(V, m), \exists\left(f_{n}\right)_{n \in \mathbb{N}} \in \mathcal{C}_{c}(V), f_{n} \rightarrow f\right.$ and $\Delta f_{n}$ converge $\}$

- $\bar{\Delta} f:=\lim _{n \rightarrow \infty} \Delta f_{n}, \quad f \in D(\bar{\Delta})$ and $\left(f_{n}\right)_{n} \in \mathcal{C}_{c}(V)$ such that $f_{n} \rightarrow f$.

For an unbounded operator the relation between the spectrum and the numerical range is more complicated. But for a closed operator we have the following inclusion, see [Kat76] and [AZ10].

Proposition 3.2. Let $T$ be a closed operator. Then $\sigma_{\text {ess }}(T) \subset \overline{W(T)}$.

More precisely, let us define the following numbers:

$$
\begin{array}{cl}
\eta(T)=\inf \{\mathcal{R} e \lambda: & \lambda \in \sigma(T)\} . \\
\nu(T)=\inf \{\mathcal{R} e \lambda: & \lambda \in W(T)\} . \\
\eta^{e s s}(T)=\inf \{\mathcal{R} e \lambda: & \left.\lambda \in \sigma_{\text {ess }}(T)\right\} .
\end{array}
$$

The Proposition 3.2 induces this Corollary.

Corollary 3.1.

$$
\eta^{e s s}(\bar{\Delta}) \geq \nu(\bar{\Delta})
$$

Remark 3.1. If $\Delta$ is self-adjoint, then $\eta(\Delta)=\nu(\Delta)$. But this is not the case in general. 
3.2. Cheeger inequalities. For a non symmetric graph $G$, we prove bound estimates on the real part of the numerical range of $\Delta$ in terms of the Cheeger constant. We use this estimation to characterize the absence of the essential spectrum of $\bar{\Delta}$.

First, we recall the definitions of the Cheeger constants on $\Omega \subset V$ :

$$
h(\Omega)=\inf _{\substack{U \subset \Omega \\ \text { finite }}} \frac{b\left(\partial_{E} U\right)}{m(U)}
$$

and

$$
\tilde{h}(\Omega)=\inf _{\substack{U \subset \Omega \\ \text { finite }}} \frac{b\left(\partial_{E} U\right)}{\beta^{+}(U)}
$$

where for a subset $U$ of $V$,

$$
\begin{gathered}
b\left(\partial_{E} U\right)=\sum_{(x, y) \in \partial_{E} U} b(x, y) \\
\beta^{+}(U)=\sum_{x \in U} \beta^{+}(x) \text { and } m(U)=\sum_{x \in U} m(x) .
\end{gathered}
$$

We define in addition:

$$
\begin{gathered}
m_{\Omega}=\inf \left\{\frac{\beta^{+}(x)}{m(x)}, \quad x \in \Omega\right\} \\
M_{\Omega}=\sup \left\{\frac{\beta^{+}(x)}{m(x)}, \quad x \in \Omega\right\} .
\end{gathered}
$$

Cheeger's Theorems had appeared in many works on symmetric graphs. They give estimations of the bottom of the spectrum of the Laplacian in terms of the Cheeger constant. The inequality (4) controls the lower bound of the real part of $\lambda \in W\left(\Delta_{\Omega}^{D}\right)$.

Theorem 3.1. Let $\Omega \subset V$, the bottom of the real part of $W\left(\Delta_{\Omega}^{D}\right)$ satisfies the following inequalities:

$$
\frac{h^{2}(\Omega)}{8} \leq M_{\Omega} \nu\left(\Delta_{\Omega}^{D}\right) \leq \frac{1}{2} M_{\Omega} h(\Omega)
$$

Proof:

From the works of J. Dodziuk [Dod06] and A. Grigoryan Gri11, we can deduce the following bounds of the symmetric quadratic form $Q_{\Delta_{\Omega}^{D}}$ on $\mathcal{C}_{c}(\Omega)$,

$$
\frac{h^{2}(\Omega)}{8} \leq M_{\Omega} \inf _{\|f\|_{m}=1} Q_{\Delta_{\Omega}^{D}}(f) \leq \frac{1}{2} M_{\Omega} h(\Omega) .
$$

Then using the equality (11) we conclude our estimation.

We deduce in particular the following inequalities. 
Corollary 3.2. Let $\Omega \subset V$, we have

$$
\frac{\tilde{h}^{2}(\Omega)}{8} \leq \nu\left(\tilde{\Delta}_{\Omega}^{D}\right) \leq \frac{1}{2} \tilde{h}(\Omega) .
$$

Proposition 3.3. Let $\Omega \subset V$ and $g \in \mathcal{C}_{c}(\Omega),\|g\|_{m}=1$. Let $\lambda=\left(\Delta_{\Omega}^{D} g, g\right)_{m} \in$ $W\left(\Delta_{\Omega}^{D}\right)$. Then

$$
m_{\Omega} \frac{\mathcal{R} e\left(\tilde{\Delta}_{\Omega}^{D} g, g\right)_{\beta^{+}}}{(g, g)_{\beta^{+}}} \leq 2 \mathcal{R} e(\lambda) \leq M_{\Omega} \frac{\mathcal{R} e\left(\tilde{\Delta}_{\Omega}^{D} g, g\right)_{\beta^{+}}}{(g, g)_{\beta^{+}}} .
$$

Proof:

We have for all $x \in \Omega$

$$
m_{\Omega} m(x) \leq \beta^{+}(x) \leq M_{\Omega} m(x)
$$

therefore

$$
m_{\Omega}(g, g)_{m} \leq(g, g)_{\beta^{+}} \leq M_{\Omega}(g, g)_{m}
$$

which implies that:

$$
m_{\Omega} \frac{\mathcal{R} e\left(\tilde{\Delta}_{\Omega}^{D} g, g\right)_{\beta^{+}}}{(g, g)_{\beta^{+}}} \leq \frac{Q_{\Delta_{\Omega}^{D}}(g)}{2(g, g)_{m}} \leq M_{\Omega} \frac{\mathcal{R} e\left(\tilde{\Delta}_{\Omega}^{D} g, g\right)_{\beta^{+}}}{(g, g)_{\beta^{+}}}
$$

because $\left(\Delta_{\Omega}^{D} g, g\right)_{m}=\left(\tilde{\Delta}_{\Omega}^{D} g, g\right)_{\beta^{+}}$, for all $g \in \mathcal{C}_{c}(\Omega)$.

Corollary 3.3. Let $\Omega \subset V$, we have

$$
m_{\Omega} \frac{\tilde{h}^{2}(\Omega)}{8} \leq \nu\left(\Delta_{\Omega}^{D}\right)
$$

We can also estimate the real part of any element of the numerical range of $\Delta_{\Omega}^{D}$ in terms of the isoperimetric constant $\tilde{h}$.

Corollary 3.4. For all $\Omega \subset V$ and $\lambda \in W\left(\Delta_{\Omega}^{D}\right)$ we have

$$
m_{\Omega}\left(2-\sqrt{4-\tilde{h}^{2}(\Omega)}\right) \leq 2 \mathcal{R} e(\lambda) \leq M_{\Omega}\left(2+\sqrt{4-\tilde{h}^{2}(\Omega)}\right) .
$$

Proof:

We follow the same approach as Fujiwara in Proposition 1 Fuj96, and we apply it to the symmetric Laplacian $\tilde{H}_{\Omega}^{D}=$ $\tilde{\Delta}_{\Omega}^{D}+\tilde{\Delta}_{\Omega}^{D}$, we obtain, for all $g \in \mathcal{C}_{c}(\Omega)$

$$
2-\sqrt{4-\tilde{h}^{2}(\Omega)} \leq \frac{2 \mathcal{R} e\left(\tilde{\Delta}_{\Omega}^{D} g, g\right)_{\beta^{+}}}{(g, g)_{\beta^{+}}} \leq 2+\sqrt{4-\tilde{h}^{2}(\Omega)} .
$$

Hence we obtain the result by a direct corollary of the inequality (5). 
3.3. Absence of essential spectrum from Cheeger constant. This subsection is devoted to the study of the essential spectrum relative to the geometry of the weighted graph. We evaluate the interest of the study of the numerical range of non self-adjoint operators. Indeed, the knowledge of the numerical range of the Laplacian brings an essential information on its essential spectrum.

We provide the Cheeger inequality at infinity on a filtration of graph $G$.

Definition 3.3. A graph $H=\left(V_{H}, \vec{E}_{H}\right)$ is called a subgraph of $G=\left(V_{G}, \vec{E}_{G}\right)$ if $V_{H} \subset V_{G}$ and $\vec{E}_{H}=\left\{(x, y) ; x, y \in V_{H}\right\} \cap \vec{E}_{G}$.

Definition 3.4. A filtration of $G=(V, \vec{E})$ is a sequence of finite connected subgraphs $\left\{G_{n}=\left(V_{n}, \vec{E}_{n}\right), \quad n \in \mathbb{N}\right\}$ such that $G_{n} \subset G_{n+1}$ and:

$$
\bigcup_{n \geq 1} V_{n}=V \text {. }
$$

Let $G$ be an infinite connected graph and $\left\{G_{n}, n \in \mathbb{N}\right\}$ a filtration of $G$. Let us denote

$$
\begin{aligned}
& m_{\infty}=\lim _{n \rightarrow \infty} m_{V_{n}^{c}} \\
& M_{\infty}=\lim _{n \rightarrow \infty} M_{V_{n}^{c}}
\end{aligned}
$$

The Cheeger constant at infinity is defined by:

$$
h_{\infty}=\lim _{n \rightarrow \infty} h\left(V_{n}^{c}\right) .
$$

Remark 3.2. These limits exist in $\mathbb{R}^{+} \cup\{\infty\}$ because $m_{V_{n}^{c}}, M_{V_{n}^{c}}$ and $h\left(V_{n}^{c}\right)$ are monotone sequences.

Remark 3.3. The Cheeger constant at infinity $h_{\infty}$ is independent of the filtration. Indeed it can be defined, as in Fuj96 and [Kel10], by $h_{\infty}=\lim _{K \rightarrow G} h\left(K^{c}\right)$, where $K$ runs over all finite subsets because the graph is locally finite.

Definition 3.5. $G$ is called with heavy ends if $m_{\infty}=\infty$.

Lemma 3.1. For any subset $\Omega$ of $V$ such that $\Omega^{c}$ is finite, we have

$$
\nu\left(\bar{\Delta}_{\Omega}^{D}\right)=\nu\left(\Delta_{\Omega}^{D}\right) .
$$

Proof:

It is easy to see that

$$
b=\inf _{\lambda \in W\left(\bar{\Delta}_{\Omega}^{D}\right)} \mathcal{R} e(\lambda) \leq \inf _{\lambda \in W\left(\Delta_{\Omega}^{D}\right)} \mathcal{R} e(\lambda)=a .
$$

Let $f \in D\left(\bar{\Delta}_{\Omega}^{D}\right)=\left\{f \in D(\bar{\Delta}), f(x)=0, \forall x \in \Omega^{c}\right\}$ such that $\|f\|_{m}=1$. Hence there is a sequence $\left(f_{n}\right) \in \mathcal{C}_{c}(V)=D(\Delta)$ which converges to $f$ and $\left(\Delta f_{n}\right)$ converges to $\bar{\Delta} f$. It follows that $g_{n}=\mathbf{1}_{\Omega} f_{n}=0$ on $\Omega^{c}$ and $\left(\Delta_{\Omega}^{D} g_{n}\right)$ converges to $\bar{\Delta}_{\Omega}^{D} f$. So

$$
a \leq \nu\left(\Delta_{U}^{D}\right) \leq \mathcal{R} e\left(\Delta_{U}^{D} g_{n}, g_{n}\right)_{m} \underset{n \rightarrow \infty}{\longrightarrow} \mathcal{R} e\left(\bar{\Delta}_{U}^{D} f, f\right)_{m}
$$

then

$$
a \leq b
$$


Theorem 3.2. The essential spectrum of $\bar{\Delta}$ satisfies:

$$
\frac{h_{\infty}^{2}}{8} \leq M_{\infty} \eta^{e s s}(\bar{\Delta})
$$

and

$$
m_{\infty} \frac{\tilde{h}_{\infty}^{2}}{8} \leq \eta^{e s s}(\bar{\Delta})
$$

Proof:

Let $\left\{G_{n}, n \in \mathbb{N}\right\}$ be a filtration of $G$, from the inequality (3) we get,

$$
\nu\left(\bar{\Delta}_{V_{n}^{c}}^{D}\right) \leq \eta^{e s s}\left(\bar{\Delta}_{V_{n}^{c}}^{D}\right) .
$$

From Theorem 5.35 of T. Kato page 244 Kat76, the essential spectrum is stable by a compact perturbation, we obtain

$$
\sigma_{e s s}(\bar{\Delta})=\sigma_{e s s}\left(\bar{\Delta}_{V_{n}^{c}}^{D}\right) \text {. }
$$

Therefore

$$
\nu\left(\Delta_{V_{n}^{c}}^{D}\right) \leq \eta^{e s s}(\bar{\Delta}),
$$

we use Theorem 3.1 and the equality (6), then we find the result by taking the limit at $\infty$.

The following Corollary follows from Theorem 3.2, It gives an important characterization for the absence of the essential spectrum especially it includes the case of rapidly branching graphs.

Corollary 3.5. The essential spectrum of $\bar{\Delta}$ on a heavy end graph $G$ with $\tilde{h}_{\infty}>0$ is empty.

Proof:

The emptiness of the essential spectrum for $\bar{\Delta}$ on a graph with heavy ends is an immediate Corollary of the inequality (8), then if $m_{\infty}=\infty$ where $\tilde{h}_{\infty}>0$, we have $\sigma_{\text {ess }}(\bar{\Delta})=\emptyset$.

Acknowledgments: I take this opportunity to express my gratitude to my thesis directors Colette Anné and Nabila Torki-Hamza for all the fruitful discussions, helpful suggestions and their guidance during this work. This work was financially supported by the "PHC Utique" program of the French Ministry of Foreign Affairs and Ministry of higher education and research and the Tunisian Ministry of higher education and scientific research in the CMCU project number 13G1501 "Graphes, Géométrie et théorie Spectrale". Also I like to thank the Laboratory of Mathematics Jean Leray of Nantes (LMJL) and the research unity (UR/13ES47) of Faculty of Sciences of Bizerta (University of Carthage) for their financial and their continuous support. I would like to thank the anonymous referee for the careful reading of my paper and the valuable comments and suggestions. 


\section{REFERENCES}

[AT15] C. Anné, N. Torki-Hamza The Gauss-Bonnet operator of an infinite graph. Anal. Math. Phys. 5, (2015), 137-159.

[AZ10] Y. Arlinskii, V. Zagrebnov. Numerical range and quasi-sectorial contractions. J. Math. Anal. Appl. 366, (2010), 33-43.

[Bal16] M. Balti On the eigenvalues of weighted directed graphs. Complex analysis and operator theory, 11, (2017), 1387-1406.

[Ber64] S. K. Berberian. The numerical range of a normal operator. Journal of the European Mathematical Society, 31, (1964), 479-483.

[DHK09] M. Demuth, M. Hansmann, G. Katriel. On the discrete spectrum of non-selfadjoint operators. J. Funct. Anal. 257, (2009), 2742-2759.

[Dod06] J. Dodziuk. Elliptic operators on infinite graphs. World Sci. Publ., Hackensack, (2006), 353-368.

[DL79] H. Donnelly, P. Li. Pure point spectrum and negative curvature for noncompact manifolds. Duke Math J, 46, (1979), 497-503.

[ELZ83] W. D. Evans, R. T. Lewis, A. Zettl. Non self-adjoint operators and their essential spectra. In form local times to global geometry, control and physics, D. Ellworthy, ed., Differential Equation and Operators, Lecture Notes in Mathematics, 1032, (1983), 123-160.

[FLS11] R. L. Frank, A. Laptev, R. Seiringer. A sharp bound on eigenvalues of Schrödinger operators on the half-line with complex-valued potentials. Spectral theory and analysis, Oper. Theory Adv. Appl. 214, (2011), 39-44.

[Fuj96] K. Fujiwara. The Laplacian on rapidly branching trees. Duke Mathematical Journal. 83, (1996), 191-202.

[GC05] L. Golinskii, S. S. Capizzano. The asymptotic properties of the spectrum of non symmetrically perturbed Jacobi matrix sequences, Journal of Approximation Theory, 144, (2005), 84-102.

[Gri11] A. Grigoryan. Analysis on graphs, Lecture Notes, University of Bielefeld, WS $(2011 / 12)$.

[Han11] M. Hansmann. An eigenvalue estimate and its application to non-selfadjoint Jacobi and Schrödinger operators, Lett. Math. Phys. 98, (2011), 79-95.

[JY12] K. Jahedi, B. Yousefi. Numerical Ranges of operators acting on Banach spaces. Czechoslovak Mathematical Journal. 62, (2012), 495-503.

[Kat76] T. Kato. Perturbation theory for linear operators. Springer-Verlag, Berlin, Heidelburg and New York, (1976).

[Kel10] M. Keller. The essential spectrum of the Laplacian on rapidly branching tessellations. Mathematische Annalen 346, (2010), 51-66. 
[Lew79] R. T. Lewis. Applications of a comparison for quasi-accretive operators in a Hilbert space. In: Everitt W., Sleeman B. (eds) Ordinary and Partial Differential Equations. Lecture Notes in Mathematics. Springer, Berlin, Heidelberg. 964, (1982), 422-434.

[T-H10] N. Torki-Hamza Laplaciens de graphes infinis. I: Graphes métriquement complets. Confluentes Math. 2, (2010), 333-350.

Translated to: Essential self-adjointness for combinatorial Schrödinger operators IMetrically complete graphs. arXiv:1201.4644v1.

[Tr05] L. N. Trefethen. Spectra and pseudospectra, the behavior of non normal matrices and operators. Princeton University Press, (2005).

Université de Carthage, Faculté des Sciences de Bizerte: Mathématiques et Applications (UR/13ES47) 7021-Bizerte (Tunisie), Université de Nantes, Laboratoire de Mathématique Jean Lauray, CNRS, Faculté des Sciences, BP 92208, 44322 NANTES, (FRANCE).

E-mail address: balti-marwa@hotmail.fr 